\title{
CT chest under general anesthesia: pulmonary, anesthetic and radiologic dilemmas
}

\author{
Mohamed Mahmoud • Christopher Towe • \\ Robert J. Fleck
}

Received: 6 October 2014 /Revised: 21 October 2014 / Accepted: 19 November 2014 / Published online: 20 December 2014

(C) Springer-Verlag Berlin Heidelberg 2014

\section{Introduction}

Today's practice of medicine involves ever more complex patients whose care is coordinated with multidisciplinary teams. Caring for these patients can challenge all members of the health care team. Sedation/anesthesia in infants/toddlers as well as uncooperative or intellectually or emotionally impaired children who require imaging studies of the chest are ongoing challenges. High-quality computed tomography (CT) chest imaging studies in children under general anesthesia are extremely important for accurate interpretation and subsequent medical decision-making. Anesthesia-induced atelectasis may obscure or mimic true pathology creating a significant quality issue. Obtaining a high-quality, motion-free chest imaging study in infants and children under general anesthesia remains a difficult task in many institutions. Meticulous attention to anesthesia and imaging techniques and specialized knowledge are required to properly perform and interpret chest imaging studies.

In this commentary, we discuss the continuous struggle to obtain high-quality CT chest imaging under general anesthesia. We will also discuss the major concerns of the

\footnotetext{
M. Mahmoud ( $\square)$

Department of Anesthesiology,

Cincinnati Children's Hospital Medical Center,

3333 Burnet Avenue, MLC 2001,

Cincinnati, OH 45229, USA

e-mail: mohamed.mahmoud@cchmc.org

C. Towe

Department of Pulmonary Medicine, Cincinnati Children's Hospital Medical Center, Cincinnati, OH, USA

R. J. Fleck

Department of Radiology,

Cincinnati Children's Hospital Medical Center,

Cincinnati, OH, USA
}

anesthesiologist, radiologist and pulmonologist and why cooperation and coordination among these providers are critical for an optimal quality study.

\section{Pulmonary concerns}

Simply put, CT imaging of the chest is needed when more routine imaging modalities, such as the standard chest radiograph, are insufficient for either the diagnosis or management of children with respiratory disease. Given the inherent risks, the decision to pursue chest CTs in children is rarely undertaken lightly, especially when sedation/anesthesia will be required for an optimal study. Therefore, when inadequate images are obtained to answer the clinical question, parents and clinicians are equally frustrated.

Chest CT is superior to conventional chest radiographs because of its ability to provide three-dimensional anatomical orientation, which allows for no overlap of anatomy and excellent resolution of differences in tissue densities. For infants, it is frequently indicated in the evaluation of suspected interstitial lung diseases or congenital anomalies [1]. In some cases of interstitial lung diseases, typical presentations of neuroendocrine cell hyperplasia of infancy for example, a properly performed chest $\mathrm{CT}$ is diagnostic [2], thus alleviating the need for more invasive procedures like surgical lung biopsy. Even when surgical lung biopsy is necessary, prebiopsy chest CT highlights areas of greatest pathological involvement increasing the likelihood of diagnosis.

In cases of suspected mediastinal or congenital anomalies, the three-dimensional images provided by chest $\mathrm{CT}$ allow for both diagnosis and presurgical planning, if necessary. Vascular anomalies such as vascular rings or vascular slings can be suggested by other imaging modalities such as contrast esophagram, but a chest CT is usually performed to confirm the diagnosis and delineate the precise anatomy or associated 
findings. Young patients with recurrent diagnoses of pneumonia frequently undergo chest $\mathrm{CT}$ scans evaluating for findings suggesting chronic aspiration, mediastinal masses, lymphadenopathy or congenital thoracic malformations such as bronchogenic cysts or congenital pulmonary airway malformation (CPAM). These are difficult to see and distinguish on chest radiograph but readily identifiable by $\mathrm{CT}$.

Because chest $\mathrm{CT}$ is frequently obtained in patients with possible chronic or congenital diagnoses, care is needed to reduce the risk of concurrent processes or artifact distorting or obscuring the diagnosis. Atelectasis is frequently encountered and should be prevented if possible. Acute processes, such as an upper or lower respiratory tract infection, can also introduce distortion, and if possible the CT scan should be deferred until the infection has been adequately treated and cleared. This is especially critical when the indication for chest CT is evaluating for an underlying congenital anomaly as the cause of the pneumonia. Artifact from overlying wires and the patient's arms can cause beam hardening and streak artifacts that can be prevented by removal of wires and proper positioning of arms.

After a diagnosis has been made, chest $\mathrm{CT}$ is sometimes indicated to follow lung disease progression or response to therapeutic interventions. While pulmonary function testing is the mainstay for monitoring respiratory diseases, it is sometimes inadequate or unavailable. Toddlers are frequently too young for conventional spirometry but too large for infant pulmonary function testing and represent an especially challenging group to monitor. Chest $\mathrm{CT}$ is sometimes utilized in these patients because it can demonstrate significant disease burden before decreases in pulmonary function are apparent. What level of chest CT-defined disease burden is clinically significant is controversial and outside the scope of this commentary.

From a pulmonary standpoint, the techniques and medications used to anesthetize/sedate and ventilate a child undergoing chest CT are dependent on the clinical situation. Safety is of paramount importance, and if an appropriate scan cannot be obtained safely, then other modalities must be explored. In most situations, the pathology of interest is at or distal to the level of the carina allowing any appropriate artificial airway to be placed without the risk of introducing distortion artifact. In certain situations, the larynx or trachea is of prime interest, and in these situations an anesthesia plan that does not involve endotracheal intubation is necessary.

\section{Anesthetic concerns}

Anesthesiologists are becoming increasingly involved in providing sedation and/or anesthesia for children undergoing medical imaging procedures. The challenge lies in balancing technical factors governing the image quality of the study with safety concerns stemming from risks related to anesthetizing a child in an off-site location like a radiology department. Providing anesthesia in a location remote to the operating room is always a challenge to anesthesiologists since support from colleagues in the operating room and time to respond to an emergent situation can be long. For patient safety, a less-ismore approach is often used. This often conflicts with the need to find an anesthetic approach that provides immobility during imaging, anxiolysis/amnesia and a rapid recovery. A variety of sedation and anesthetic techniques are used to facilitate imaging studies with none being the standard of care. If resources are available, most anesthesiologists elect to use general anesthesia rather than deep sedation for CT scanning; some may choose endotracheal intubation or laryngeal mask airway (LMA) while others may choose to maintain the airway only with oxygen delivered by nasal cannula with periodic use of one or more inhalational or intravenous anesthetic agents. In general, there is a tendency to avoid intubating a child for CT unless there is a clear requirement for intubation.

Perhaps the most important cause of a suboptimal study is the lack of in-depth understanding of the anesthetic requirement for the different CT chest image studies. Understanding the goal of each imaging study allows the anesthesiologist to choose the safest and most effective regimen to allow a meaningful study. As an example, the goal of dynamic airway imaging in patients who cannot cooperate with breathing instructions is to evaluate airway changes during spontaneous respiration. Utilizing an inhalational agent such as sevoflurane or intravenous propofol is safe and has the advantage of fast induction and recovery times. When a CT scan is performed to evaluate lung parenchymal detail (a high-resolution $\mathrm{CT}$ ), motion-free images at full inspiration and usually at expiration are required. These requirements cannot be met with spontaneous respiration and would likely lead to a study compromised by atelectasis. Effective communication, through discussion between the anesthesiologist and radiologist or a detailed and clear written protocol, allows the anesthesia team to choose the anesthetic technique required for each CT chest imaging examination.

A second important reason for a poor-quality $\mathrm{CT}$ chest study is the lack of appreciation of how much lung atelectasis can be associated with general anesthesia in the absence of a standardized anesthesia, lung recruitment and controlledventilation technique. Infants and children are prone to atelectasis under general anesthesia due to high chest wall compliance and underdeveloped collateral ventilation system coupled with the loss of respiratory drive and loss of intercostal muscle tone. In the very young patient, closing volume tends to increase and functional residual capacity decrease during general anesthesia due to cephalad displacement of the most dorsal part of the diaphragm, with impaired ventilation of the inferior and dependent parts of the lungs resulting in atelectasis [3]. Bendixen and colleagues [4] showed that 
spontaneous breathing without periodic deep breaths can lead to progressive atelectasis, with increased shunting and decreased pulmonary compliance, and these changes were reversible by lung hyperinflation.

Atelectasis in the dependent regions of lungs appears within 5 min of induction of anesthesia [5], which is why it is extremely important to use a tight-fitting face mask during anesthesia induction and to add continuous positive airway pressure (CPAP) as soon as consciousness is lost. If spontaneous breathing is allowed without immediate CPAP, the battle against atelectasis will be lost early and full recovery of the atelectatic portions can be difficult. It is important to note that atelectasis can develop with both inhalational and intravenous anesthesia and whether the patient is spontaneously breathing or is paralyzed and mechanically ventilated [6].

In healthy lungs, collapsed areas are re-expanded, and a normal functional residual capacity restored if airway pressures are raised beyond the alveolar opening pressure. Bendixen et al. [4] and later Rothen et al. [7] showed that this pressure is approximately $40 \mathrm{~cm} \mathrm{H}_{2} \mathrm{O}$. Lachmann et al. [8] recommended opening collapsed lungs by applying sufficient levels of peak inspiratory pressure and maintaining alveoli open by using sufficient levels of positive end-expiratory pressure (PEEP). This recruitment maneuver has been shown to improve arterial oxygenation and lung compliance [9]. Tusman et al. [9] showed that the frequency of atelectasis was much less following the alveolar recruitment strategy peak airway pressure of $40 \mathrm{~cm} \mathrm{H}_{2} \mathrm{O}$ and a PEEP of $15 \mathrm{~cm}$ $\mathrm{H}_{2} \mathrm{O}$ for 10 breaths. PEEP was then reduced to and kept at $5 \mathrm{~cm} \mathrm{H}_{2} \mathrm{O}$. Compared with children who did not have the maneuver performed, the mere application of $5 \mathrm{~cm} \mathrm{H}_{2} \mathrm{O}$ of CPAP without a prior recruitment did not show the same treatment effect and showed no difference compared to the control group without PEEP [10]. It is also important to note that while using high inflating pressures is benign in most cases, high-pressure ventilatory strategies can lead to hemodynamic compromise and possibly cause lung injury from over-distension of non-collapsed lung areas [11, 12]. The possibility of these risks from high airway pressures has to be balanced against the benefit of the study. For example, the risks of applying these recruiting maneuvers are far greater in a child with cardiomyopathy and recent pneumothorax than in an outpatient with suspected tracheomalacia. The imaging and anesthesia protocols need to be discussed extensively among all providers before anesthesia induction. In this case, transient respiratory pauses long enough to acquire motion-free CT images of the lungs induced in a sedated infant or young child by synchronously applying positive pressure with face-mask ventilation to several consecutive spontaneous tidal inspirations might provide adequate images at much lower risk.

A third anesthetic concern is the use of LMA for CT chest imaging. LMA is increasingly being used in children as it is less invasive than endotracheal intubation and causes less discomfort in the recovery period. One of the limitations of using LMA in children is that its low-pressure seal is often inadequate for positive pressure ventilation with PEEP. It is impossible to provide alveolar recruitment strategy with peak airway pressure of $40 \mathrm{~cm} \mathrm{H}_{2} \mathrm{O}$ when classic LMA is used. The ProSeal laryngeal mask airway (PLMA) (Laryngeal Mask Co., Henley-on-Thames, U.K.) is a relatively new supraglottic airway device with a drain tube to minimize the risk of gastric insufflation and aspiration. Although the PLMA has been shown to form a more effective seal than the classic LMA [13], obtaining high inspiratory pressures with an LMA remains very difficult.

The final concern is moving a patient into the prone position required to re-expand dependent atelectasis and to clarify the nature of dependent lung opacities. Moving a patient into the prone position can be a challenge to anesthesia providers and radiology technicians especially in obese children/adolescents. Prone position is associated with predictable changes in physiology including an increase in functional residual capacity, but it can also be associated with a variety of complications [14]. Reduction in cardiac index in prone position is attributed to reduced venous return, direct effects on arterial filling and reduced left ventricular compliance secondary to increased thoracic pressure. Other risks include dislodging the airway, vascular access or monitoring equipment.

\section{Radiology concerns}

To a radiologist reading chest $\mathrm{CT}$ in children, atelectasis is the bane of proper interpretation and diagnosis. As such, we need to work with our colleagues in anesthesia and pulmonary medicine to come up with a better approach. Often, the first step is to create a uniform approach among anesthesiologists and radiologists so that it is easiest to communicate a plan for each individual patient

Each patient and indication has its own challenges. For example, high-resolution CT is used for the evaluation of the fine detail in the lung parenchyma and often requires inspiratory and expiratory imaging, but it is performed without contrast. Imaging with a good inspiratory and expiratory pause can be quite difficult; therefore, the choice is often made to intubate. However, the question should be asked: "Are the expiratory images really needed?" I think that review of your own imaging experience would indicate they are often not needed. In fact, some pediatric institutions have moved to only perform expiratory imaging when the pretest probability of its helpfulness is high. This allows imaging without sedation or using a feed and swaddle technique in a large percentage of cases. By avoiding general anesthesia and its inherent production of atelectasis would this result in less atelectasis? We believe that it would, but there is no evidence in the current 
literature. Certainly, avoiding the risk and cost of anesthesia is better for the patient.

Administration of contrast concomitant with CT adds another dimension to CT because the process of injection can arouse a small child from a calm or even sedated state. An example of this is a CT angiography in a patient with a congenital lung lesion. Atelectasis will cause crowding of the vessels and may obscure portions of the pathology. The presence of atelectasis when looking for a vascular ring would be less limiting but could result in missing unsuspected pathology. So shouldn't we do the best we can every time we CT the chest? Careful attention to detail has allowed performance of contrast-enhanced $\mathrm{CT}$ in non-sedated/general anesthesia young children with excellent results.

A third challenge is young patients with malignancy that need evaluation for lung metastases. Many of these patients are at an age where they do not need sedation, but they have atelectasis from their immobility due to morbidity associated with treatment. Others can hold still enough despite their young age for a brief helical or volume CT. Those who cannot will require sedation/anesthesia. In these cases, atelectasis can obscure nodules that may change the staging and treatment. How do we deal with atelectasis due to patient immobilization and morbidity, whether they are conscious or under general anesthesia/sedation?

All important questions should be raised during protocoling for $\mathrm{CT}$ chest imaging and communicated to the anesthesia providers and CT technicians before imaging. In other words, which child should be allowed to try without sedation? Which child should only be imaged under general anesthesia with intubation and maximal inspiratory breathhold? Is a transient respiratory pause induced with sedation by synchronously applying positive pressure with face-mask ventilation to several consecutive spontaneous tidal inspirations an acceptable option for the clinical question asked? Is calm breathing by using a volume acquisition mode while the patient is sleeping after a feeding (feed and swaddle technique) a reasonable choice? Should we consider scanning some patients prone as routine - i.e. congenital lung lesions that are known to be posterior from prenatal images or preceding radiographs?

In an ideal and perfect world, all of these questions should be asked before scheduling the patient. It is extremely hard for a busy radiology practice to go through all these questions before scheduling, particularly when many cases are scheduled and rescheduled. Therefore, protocols are often performed closer to the day of the appointment and, on day of imaging, it is appropriate to readdress these questions and communicate with all personnel involved. Otherwise, these questions will be raised at the conclusion of the study, which is the wrong time to be asking questions. Once the images are acquired, the radiologist is in a tough position: repeat the scans thus doubling the radiation exposure or let it go and do the best interpretation that can be done with limited quality.

\section{Graduated approach to obtain an ideal CT chest evaluation under anesthesia}

One of the major reasons for suboptimal chest evaluation is lack of communication among all providers involved in the care of patients presented for CT evaluation. Since chest CT scans scheduled with general anesthesia are obtained in multiple different clinical situations, regular communication among the ordering provider, anesthesiologist and radiologist is very important to increase the likelihood of obtaining a clinically meaningful result. Every member of the team, before proceeding, should understand the indications and a priori differential diagnosis. In addition to the individualized communications surrounding each case, regular multidisciplinary meetings should be held to debrief outcomes and implement quality improvement measures.

In an ideal world, a designated team of anesthesiologists should be committed to providing anesthesia care and troubleshooting the logistical challenges in the radiology department. Having an anesthesiology team that is embedded, engaged, interested, educated, proactive and passionate about optimizing sedation/anesthesia for imaging ("do it once, do it right") is key, but this is not the scenario in many institutions due to limited resources. Each member of this team should be familiar with demands unique to each imaging study and communicate any concern immediately with other providers involved in the care of these patients.

Lack of standardized protocols during CT studies leads to a wide variability in the anesthetic techniques and, consequently, in results achieved. The saying "an ounce of prevention is worth a pound of cure" should encapsulate the mind-set of dealing with $\mathrm{CT}$ chest imaging under general anesthesia. A well-designed protocol with input from the anesthesia, radiology and pulmonary departments is essential to obtain an optimal CT chest evaluation under anesthesia. A recent publication by Newman et al. [15] concluded that controlledventilation infant $\mathrm{CT}$ scanning under general anesthesia, utilizing intubation and recruitment maneuvers followed by chest CT scans, appears to be a safe and effective method to obtain reliable and reproducible high-quality, motion-free and atelectasis-free chest CT images in children. This study describes how collaboration among the anesthesiology, pulmonology and radiology departments leads to developing a standardized controlled-ventilation CT technique and eventually a high-quality study [15]. CT scans with multisegmental/lobar atelectasis decreased from $40 \%$ to $14 \%$ between the non-protocol and the protocol scans, a statistically significant gain. 
Encourage pre-imaging discussion among the anesthesiologist, radiologist, pulmonologist and schedulers to explore any concerns as early as possible. As an example, if the radiologist feels that a certain anesthetic technique might negatively impact a test, he/she should voice this concern before imaging. Additionally, if the radiologist believes respiratory cessation is needed for diagnostic image quality, this is discussed with the anesthesiologist well before the study takes place.

Prevention of atelectasis during CT starts with consideration of the scheduling sequence of diagnostic tests in patients with complex care. Scheduling the chest CT prior to any other procedure requiring sedation or anesthesia is paramount. This may mean scheduling the CT prior to sedation for biopsy or anesthesia for bronchoscopy and especially MRI before the patient is bedridden or immobilized for any significant period of time. Second, consider performing the study without sedation/anesthesia by attempting the feed and swaddle technique in infants and utilizing child life specialists and their techniques to get a child to cooperate. This is most easily done if intravenous access is not needed and inspiratory/expiratory imaging is not required. However, ability to obtain an optimal chest CT during free-breathing depends on the type and speed of CT scanner available.

At our institution, we implemented a morning huddle that includes the imaging nurse facilitator, the CT technologist facilitator, anesthesiologist and a radiologist. This huddle takes place at 7:30 a.m. every weekday. During this huddle, any issues/concerns are identified and discussed to anticipate requirement concerns for $\mathrm{CT}$ imaging studies. The morning huddle did not solve all problems with communications at our institution, but at least it clarified the needs for each study.

\section{Conclusion}

A thorough understanding of the requirements of the imaging study, recognizing the effects of different anesthetic techniques on the development of atelectasis, and meticulous procedural management are essential to obtain high-quality study. Effective communication and collaboration among the anesthesiology, radiology and pulmonary departments will to provide the safest and most meaningful study. A clear standardized protocol for controlled-ventilation and lung recruitment maneuvers is highly recommended for those patients intubated and under anesthetia but may not be what every patient requires. Instead, tracking and grading the quality of the CT scans with regard to atelectasis may lead each institution to develop best practices for their institution based on anesthesia resources and type or CT scanner.

Conflicts of interest None

\section{References}

1. Kurland G, Deterding RR, Hagood JS et al (2013) An official American Thoracic Society clinical practice guideline: classification, evaluation, and management of childhood interstitial lung disease in infancy. Am J Respir Crit Care Med 188:376-394

2. Brody AS, Guillerman RP, Hay TC et al (2010) Neuroendocrine cell hyperplasia of infancy: diagnosis with high-resolution CT. AJR Am J Roentgenol 194:238-244

3. Reber A, Nylund U, Hedenstierna G (1998) Position and shape of the diaphragm: implications for atelectasis formation. Anaesthesia 53: 1054-1061

4. Bendixen HH, Hedley-Whyte J, Laver MB (1963) Impaired oxygenation in surgical patients during general anesthesia with controlled ventilation. A concept of atelectasis. N Engl J Med 269:991-996

5. Hedenstierna G, Lundquist H, Lundh B et al (1989) Pulmonary densities during anaesthesia. An experimental study on lung morphology and gas exchange. Eur Respir J 2:528-535

6. Strandberg A, Tokics L, Brismar B et al (1986) Atelectasis during anaesthesia and in the postoperative period. Acta Anaesthesiol Scand 30:154-158

7. Rothen HU, Sporre B, Engberg G et al (1993) Re-expansion of atelectasis during general anaesthesia: a computed tomography study. Br J Anaesth 71:788-795

8. Lachmann B (1992) Open up the lung and keep the lung open. Intensive Care Med 18:319-321

9. Tusman G, Bohm SH, Vazquez de Anda GF et al (1999) 'Alveolar recruitment strategy' improves arterial oxygenation during general anaesthesia. Br J Anaesth 82:8-13

10. Tusman G, Bohm SH, Tempra A et al (2003) Effects of recruitment maneuver on atelectasis in anesthetized children. Anesthesiology 98: $14-22$

11. Dambrosio M, Roupie E, Mollet JJ et al (1997) Effects of positive end-expiratory pressure and different tidal volumes on alveolar recruitment and hyperinflation. Anesthesiology 87:495-503

12. Puybasset L, Cluzel P, Gusman P et al (2000) Regional distribution of gas and tissue in acute respiratory distress syndrome. I. Consequences for lung morphology. CT Scan ARDS Study Group. Intensive Care Med 26:857-869

13. Goldmann K, Roettger C, Wulf H (2005) Use of the ProSeal laryngeal mask airway for pressure-controlled ventilation with and without positive end-expiratory pressure in paediatric patients: a randomized, controlled study. Br J Anaesth 95:831-834

14. Edgcombe H, Carter K, Yarrow S (2008) Anaesthesia in the prone position. Br J Anaesth 100:165-183

15. Newman B, Krane EJ, Gawande R et al (2014) Chest CT in children: anesthesia and atelectasis. Pediatr Radiol 44:164-172 